\title{
SAR and AIS Fusion for Maritime Surveillance
}

\author{
Lotfi Achiri, Raffaella Guida, Pasquale Iervolino \\ Surrey Space Centre \\ University of Surrey \\ Guildford, UK \\ \{L.Achiri, R.Guida, P.Iervolino\}@surrey.ac.uk
}

\begin{abstract}
This paper presents a novel approach to fuse Synthetic Aperture Radar (SAR) images and Automatic Identification System (AIS) data for maritime surveillance. The procedure consists of four steps. First, ship detection is performed in the SAR image using a Constant False Alarm Rate (CFAR) algorithm; then feature extraction (ship position, heading and size) is performed on ships detected in the SAR image, the third step consists in identifying the detected ships and extracting the same features from the AIS data. The final step is to feed the fusion block with both features vectors extracted separately from the SAR and AIS. Here the arithmetic mean function is established. The algorithm is tested using simulated SAR images and AIS data. Preliminary results of the fusion of SAR and AIS data are presented and discussed.
\end{abstract}

Keywords-SAR, AIS, ship detection, ship monitoring, data fusion, maritime surveillance.

\section{INTRODUCTION}

It is well known that the most efficient and cost-effective way of international trade can be done via sea which represents more than $80 \%$ of the global trade [1]. Therefore, ship monitoring (including detection and identification) plays a critical role to improve the security and safety at sea in maritime surveillance domain allowing, for example, the reporting of any illegal activities such as illegal fishing, illegal immigration and hijacking. SAR (Synthetic Aperture Radar), is an active microwave remote sensing radar system, that can acquire data independently from daylight and weather condition [2].

In the last decade, maritime surveillance domain has increased its importance and several new SAR platforms have been launched recently to enhance the security and safety at sea. For example, in June 2016, the successful launch of the Maritime Monitoring and Messaging Microsatellite (M3MSat) developed by the Canadian Space Agency (CSA) with the collaboration of the Defense Research and Development Canada (DRDC) is providing a potential SAR and AIS (Automatic Identification System) data for ship monitoring [3]. Similarly, in February 2018, the successful launch of Paz satellite, the SAR observation Spanish satellite developed by HisdeSAT started providing a high-resolution X-band SAR and AIS data [4]. Also, NovaSAR-S mission, a low-cost SAR satellite developed by Surrey Satellite Technology Ltd (SSTL) with the collaboration of Airbus Defense and Space (Airbus DC) and the support of the UK government will deliver S-Band SAR data for maritime applications in addition to its secondary
AIS payload [5]. The potential data availability of SAR images in S-Band and X-Band in addition to the AIS data provided from the same platform encourage the research community to investigate methods and develop algorithms to make best use of these data sets.

The AIS system was initially developed for ship collision avoidance by broadcasting the ship information to other ships and AIS base stations located along coast lines. This information can be dynamic including ship position, heading and speed or static including ship name, IMO number and size [6].

In maritime domain, several algorithms and methods have been developed for ship detection using SAR data. The most common ship detector algorithm is the CFAR (Constant False Alarm Rate) detector where the outcomes of the detection stage can be validated with the AIS data as a ground truth [7]. However, the AIS data are often not a reliable source of information because of the presence of some errors and uncertainties while reporting ships information, for example, error related to the ship position, heading or simply some data can be missing in the AIS report [8].

Because of the aforementioned reasons, we propose a novel approach where the AIS data are used as additional source of information by fusing it with SAR data to improve the security in maritime monitoring. First, the SAR image is pre-processed, then the CFAR detector is applied to generate a map of detected ships. This will be followed by extracting the ship features (the ship position, heading, length and width) from the map of detected ships. These features are then concatenated so that every ship is represented by one feature vector. The same features are considered for the AIS data. Vectors of features extracted from both sources of information are fed to the fusion block where a fusion strategy based on the arithmetic mean function is implemented [9].

This paper is organised as follows. Section II outlines the methodology and flowchart of the fusion of the SAR and AIS data. This is followed by the preliminary results of the implementation of the algorithm in section III. The conclusions and future work are discussed in section IV.

\section{Methodology}

This section outlines in detail the methodology of the proposed approach. First, the features extraction from SAR image and AIS data are presented. This is followed by fusing 
SAR and AIS feature vectors for ship monitoring in the flowchart presented in Fig. 1.

\section{A. SAR processing}

\section{1) Pre-processing}

Pre-processing consists of preparing the SAR image before applying the detection algorithms. If the input is a Single Look Complex product, pre-processing includes the calibration step where the pixel digital number is converted to a calibrated radar backscatter coefficient, the generation of the intensity or the amplitude images, speckle filtering and multi-looking to reduce the false alarm. Finally, the land masking is applied to consider only the sea pixels. The land masking step is very important because the ship detector algorithms usually produce high false rate detection over land area [10].

\section{2) CFAR detector}

The CFAR detector is the most common SAR-based ship detector. It is based on two main steps. First the sea clutter is modelled by a suitable statistical distribution. Once the sea clutter is modelled, a threshold value is set in order to have a constant false alarm rate according to [10]:

$$
P F A=1-\int_{-\infty}^{T} f(x) d x=\int_{T}^{+\infty} f(x) d x
$$

where $P F A$ is the false alarm probability, $f(x)$ is the sea clutter distribution and $T$ is the threshold value. Thus, a pixel or group of pixels are classified as ship if their intensity values are bigger than the threshold $T$.

\section{3) Feature extraction}

The feature extraction is the crucial part of the proposed method where, for each ship detected in the previous step, corresponding position, heading and size are extracted from the SAR image.

\section{a) Ship position}

The ship position $(x, y)$ is the centre of the detected ship represented by its azimuth and slant range in the SAR image. This can be extracted by isolating every detected ship in separated sub-image, identifying the smallest window of pixels covering the entire ship (and only one at a time) and extracting its centre of mass. The window centre is considered as the ship position. The coordinates of this centre are saved in the position vector.

\section{b) Ship heading}

Then, the 2-D Radon transform [11] is applied to determine the dominant heading of the ship defined by (2).

$$
\begin{gathered}
R(r, \theta)=\int_{-\infty}^{+\infty} \int_{-\infty}^{+\infty} I(x, y) \delta(r-x \cos \theta \\
-y \sin \theta) d x d y
\end{gathered}
$$

where $R($.$) is the Radon transform, I(x, y)$ is the image intensity, $\delta$ is the Dirac function, $r$ is the smallest distance from the origin of the coordinate system to the line $L_{r, \theta}$ defined by $L_{r, \theta}: r=$ $x \cos \theta-y \sin \theta, \theta$ is the angle between the $\mathrm{x}$-axis and the line

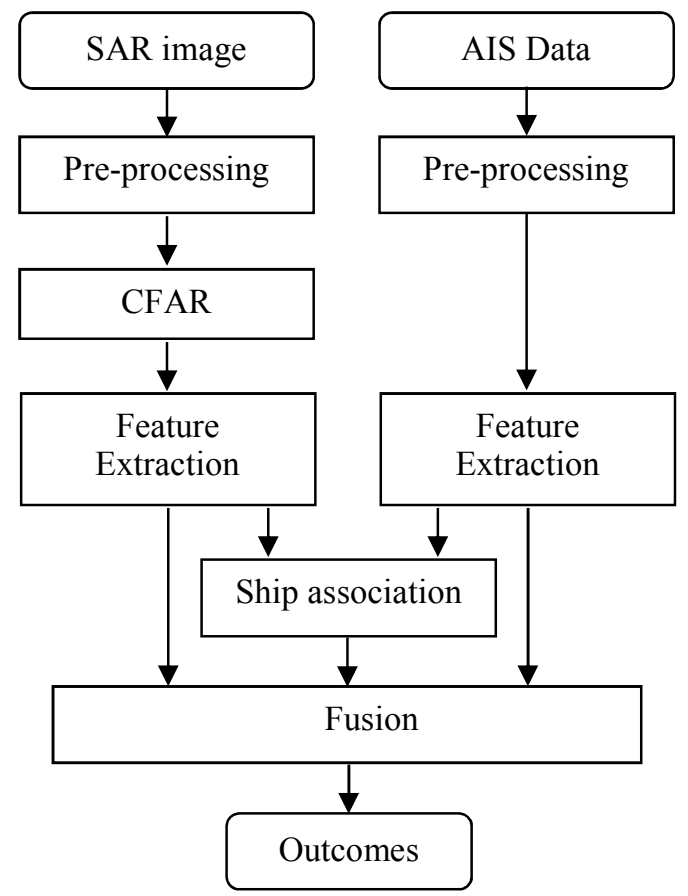

Fig. 1. The flowchart for SAR and AIS data fusion.

passing by the origin and perpendicular to $L_{r, \theta}$. The dominant orientation in the image which is the ship heading, corresponds to the highest coefficient of Radon transform. These headings are saved in the heading vector.

\section{c) Ship size}

In this paper, the ship is simplified as a rectangular shape and the length and the width of the ship can be estimated in two steps. First, the ship signature is rotated, using the heading angle extracted by Radon transform, and made parallel to y-axis. Then, the projection of the nonnull pixels over the $\mathrm{x}$-axis corresponds to the width of the ship, likewise, the projection of nonnull pixels over the y-axis corresponds the length of the ship. Both projections should be multiplied by the pixel spacing to get a value of the ship dimension in meter. Finally, the length and the width of the ship are saved in the size vector.

\section{B. AIS processing}

The AIS data are the record of the dynamic and the static information of the AIS signal broadcast by ships over a period of time. The final file of the AIS data may contain duplicated information of the same ship because the same ship could report its information more than once at the same time. Also, some information (i.e. ship name, ship length, heading) may not be provided for the monitored area. Another anomaly that may occur in AIS data are the ship position reported at zero latitude and zero longitude. Knowing the anomalies that the AIS data suffer from, a first step is removing them for the ship of interest (i.e. those detected also in the SAR image) [12].

\section{AIS and SAR fusion}

At the first stage of the development of the proposed approach, before the fusion stage, it is necessary to match the 
ships with their corresponding AIS reports. This is done by evaluating the absolute distance between the coordinates of the position of the ships detected in the SAR image and the ship positions reported in the AIS data. Here, the minimum distance method is used to find the SAR/AIS matching.

The fusion block for the SAR and AIS data is based on the arithmetic mean function [9]. The features extracted from SAR image including ship positions, heading and size (length, width) are concatenated and assembled into one vector for each ship. Same features are considered from the AIS data for every ship from the SAR image. We assume that the SAR and AIS data are acquired at the same time over a scene with low shipping density and that all ships in the SAR image are broadcasting their AIS signals. Finally, we assume that the SAR image azimuth direction is pointing to the geographic North. This last assumption is made only for sake of computation simplicity but will be relaxed in future.

\section{EXPERIMENTAL RESULTS}

The algorithm has been tested and evaluated with a simulated data sets. The SAR image is simulated in the stripmap mode with a spatial resolution of 6 meters. The size of the image is $2200 \times 3300$ pixels in azimuth and range direction respectively, consequently covering an area of $13.2 \mathrm{~km}$ by $19.8 \mathrm{~km}$. The SAR image contains 10 ships as depicted in fig.2. The exponential distribution is assumed for the sea clutter intensity (the simulated SAR data are not multi-looked here). Its mean value is set equal to that corresponding single scattering from rough sea within Geometric Optics (GO) approximation which takes the radar parameters (frequency, look angle and spatial resolution), roughness parameters and the dielectric of the saline water as an input [13]. Then, the ship backscatter is simulated using the canonical model presented in [13]. The AIS data of the 10 ships is created based on real information provided in [14]. The ship positions and headings are generated randomly. The speeds instead are the average speed recorded in [14].

After the pre-processing stage, the detection stage is performed using CFAR algorithm where the sea-clutter is modelled by exponential distribution. The $P F A$ is set equal to $10^{-5}$. The outcome of the detection stage is shown in Fig. 3. while Fig. 4. shows the effect on the image after applying the rotation to extract the ship dimensions. The projection of nonnull pixels on $y$-axis corresponds to the length of the ship, whilst the

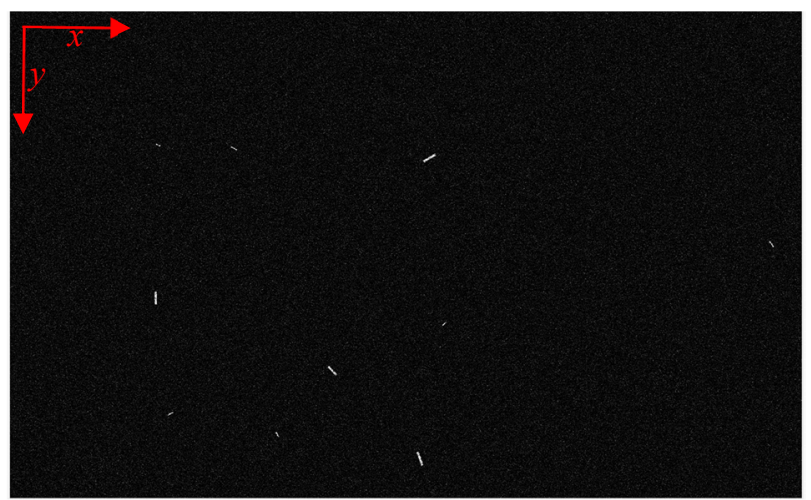

Fig. 2. Simulated SAR image intensity of the sea clutter in the stripmap mode with 10 ships.

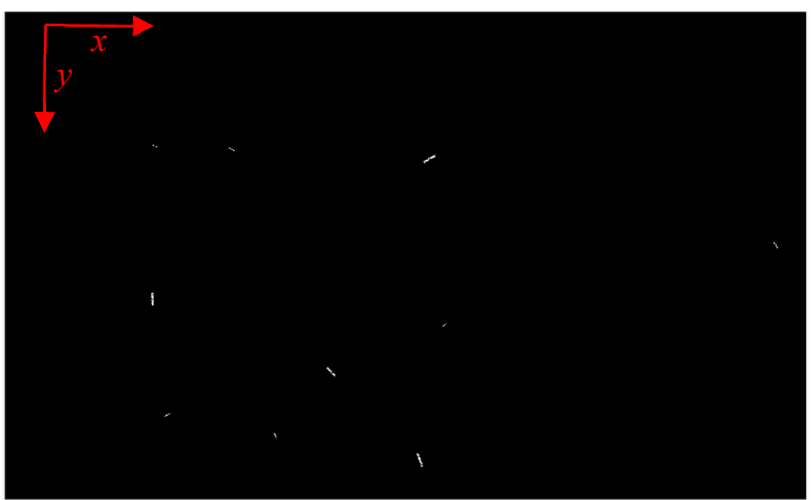

Fig. 3. The outcomes of the CFAR block represent a map of detected ships.

projection of nonnull pixels on the $\mathrm{x}$-axis corresponds to the width of the ship. As anticipated, these features extracted from the SAR simulated image together with ship position and heading are concatenated in one vector which represents an input for the fusion block.

Table 1 summarises the outcomes of the fusion block of 10 ships along with their absolute and the relative error of measurement. In the mathematical expressions of the errors, the fusion results and the AIS data are considered the measured and the reference data, respectively. The average absolute error shows a very good result in terms of ship heading with an error lower than $2^{\circ}$ and ship length $(3.27 \mathrm{~m}$ at average); while worse results are obtained in terms of ship width (7.13 $\mathrm{m}$ at average) and ship positions (3.9 and 5.9 pixels in $x$ and $y$ direction respectively). The average relative error presents a relative error of $1.77 \%$ for the ship length and a larger relative error of $24.18 \%$ for the ship width. This can be due to the width extraction technique that should be further investigated. The drawback of this method is the necessity to extract all features from both SAR and AIS sources; but unfortunately, this is not always possible (i.e. missing information in some AIS reports or impossibility to extract the ship sizes from SAR data where only one or two pixels are detected). However, using other fusing methods such us the weighted mean or the vote strategy [9] can potentially ameliorate the present results even though some data are missing from one source or another. In addition, the absence of ground truth has driven the authors to use the same AIS data for now to evaluate the error generated for each feature in the fusion block. This can be avoided if a second source of AIS data is available. In this case, the additional AIS source can be used as reference data.

Finally, the overall performance of the proposed architecture can be improved by reducing the error generated during the feature extraction stage. For instance, evaluating the azimuth

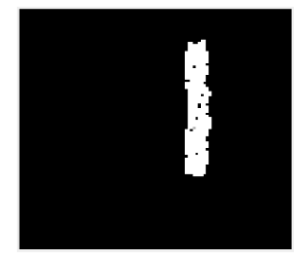

Fig. 4. Ship signature after detection with CFAR algorithm and rotation. 
TABLE I. THE FUSION RESULTS OF SAR AND AIS DATA FOR 10 SHIPS.

\begin{tabular}{|c|c|c|c|c|c|}
\hline & \multicolumn{2}{|c|}{ Position } & \multirow{2}{*}{$\begin{array}{c}\text { Heading } \\
{\left[{ }^{\circ}\right]}\end{array}$} & \multirow{2}{*}{$\begin{array}{c}\text { Length } \\
\text { [m] }\end{array}$} & \multirow{2}{*}{$\begin{array}{c}\text { Width } \\
\text { [m] }\end{array}$} \\
\hline & $x$ & $y$ & & & \\
\hline Ship 1 & 653 & 1284.5 & 178.5 & 346.5 & 54.3 \\
\hline Ship 2 & 1698.5 & 1946 & 338.5 & 399.85 & 61.2 \\
\hline Ship 3 & 1711 & 673 & 57.5 & 366 & 69.2 \\
\hline Ship 4 & 1313 & 1652 & 139 & 329.5 & 55.5 \\
\hline Ship 5 & 679.5 & 18.56 & 240.5 & 165.2 & 29.2 \\
\hline Ship 6 & 655 & 651 & 109.5 & 129.1 & 28.9 \\
\hline Ship 7 & 3132.5 & 1070 & 146.5 & 194.1 & 35.9 \\
\hline Ship 8 & 1105 & 1902 & 333 & 145.5 & 28.5 \\
\hline Ship 9 & 911 & 656.5 & 301 & 183.3 & 28.8 \\
\hline Ship 10 & 1851 & 1452.5 & 224.5 & 139.6 & 29 \\
\hline $\begin{array}{l}\text { Average of the } \\
\text { absolute error } \\
\text { over } 10 \text { ships }\end{array}$ & $\begin{array}{c}3.9 \\
\text { pixels }\end{array}$ & $\begin{array}{c}5.9 \\
\text { pixels }\end{array}$ & $1.45^{\circ}$ & $3.27 \mathrm{~m}$ & $7.13 \mathrm{~m}$ \\
\hline $\begin{array}{l}\text { Average of the } \\
\text { relative error } \\
\text { in [\%] over } 10 \\
\text { ships }\end{array}$ & - & - & - & 1.77 & 24.18 \\
\hline
\end{tabular}

displacement due to the radial velocity of the ship due to the Doppler effect can be used for a better estimation of the ship position. Preliminary results encourage the authors to test and evaluate the algorithm using real SAR and AIS data. The results of real SAR and AIS data acquired respectively from Sentinel-1 and ExactEarth provider will be presented at the conference.

\section{CONCLUSION}

The paper presents a novel approach to fuse SAR and AIS data for maritime surveillance. The fusion is performed on the decision level using the arithmetic mean function where features including ship position, heading, length and width are extracted from both data sets in order to enhance the ship monitoring operation. The approach has been tested on simulated data sets and with the assumption that all ships in the SAR image broadcast their AIS data with no temporal gap between them. Preliminary results are very promising for the fusion of SAR and AIS data for ship monitoring. Among other things, it is necessary to evaluate the Doppler shift due to the radial velocity of the ship in order to improve the current findings and the technique in general. The authors are now investigating complex scenarios on both simulated and real data sets and comparing different fusion methods.

\section{REFERENCES}

[1] Internatinal Marine Organization, "Introduction to IMO," Internet: http://www.imo.org/en/About/Pages/Default.aspx, [28 April 2018].

[2] F. T. Ulaby et al., Microwave Radar and Radiometric Remote Sensing, Ann Arbor, MI, USA:Univ. Michigan Press, 2014.

[3] Canadian Space Agency, "Maritime Monitoring and Messaging Microsatellite (M3MSat), " Internet: http:// www.asccsa.gc.ca/eng/satellites/m3msat/default.asp, [24 April 2018]

[4] B. Gomez Miguel et al., "PAZ SAR System Validation Plan," EUSAR 2014; 10th European Conference on Synthetic Aperture Radar, Berlin, Germany, 2014, pp. 1-4.

[5] R. Bird, P. Whittaker, K. Hall, C. Slim and M. Cohen, "NovaSAR: Next generation developments," 2015 IEEE 5th Asia-Pacific Conference on Synthetic Aperture Radar (APSAR), Singapore, 2015, pp. 94-98.

[6] International Telecommunication Union, "Technical characteristics for an automatic identification system using time division multiple access in the VHF maritime mobile frequency band", internet: http:// www. itu.int/dms pubrec/itu-r/rec/m/R-REC-M.1371-5-201402-I!!PDF-E.pdf, [27 October 2017].

[7] R. Pelich, N. Longépé, G. Mercier, G. Hajduch and R. Garello, "AISBased valuation of Target Detectors and SAR Sensors Characteristics for Maritime Surveillance," in IEEE Journal of Selected Topics in Applied Earth Observations and Remote Sensing, vol. 8, no. 8, pp. 3892-3901, Aug. 2015.

[8] A. Harati-Mokhtari, A. Wall, P. Brooks, J. Wang "Automatic identification system (AIS): Data reliability and human error implications" Journal of Navigation. vol. 60, no. 3, pp. 373-389, 2007.

[9] C. A. Shipp, L. I. Kuncheva, Relationships between combination methods and measures of diversity in combining classifiers, Information Fusion, vol. 3, no. 2, 2002, pp. $135-148$.

[10] D. J. Crisp, "The state-of-art in ship detection in synthetic aperture radar imagery," Aust. Gov., Dept. Def., Def. Sci. Technol. Org. (DSTO)., Australia, Tech. Rep. DSTO-RR-0272, 2004.

[11] S. Tabbone, L. Wendling, J.P. Salmon "A new shape descriptor defined on the Radon transform" Computer Vision and Image Understanding, vol. 102, no. 1, pp. 42-51, 2006.

[12] J. English, R. Hewitt, D. Power and J. Tunaley, "ICE-SAIS - Spacebased AIS and SAR for improved Ship and Iceberg Monitoring," 2013 IEEE Radar Conference (RadarCon13), Ottawa, ON, 2013, pp. 1-6.

[13] P. Iervolino and R. Guida, "A Novel Ship Detector Based on the Generalized-Likelihood Ratio Test for SAR Imagery," in IEEE Journal of Selected Topics in Applied Earth Observations and Remote Sensing, vol. 10, no. 8, pp. 3616-3630, Aug. 2017.

[14] "Maritime traffic", Internet: www. marinetraffic.com, [24 April 2018]. 